Nitschack, H. - Macunaíma, ein Wilhelm Meister in den Tropen?

\title{
Tropische Subjektivität und europäische Bildungstradition: Macunaíma, der Held ohne jeden Charakter von Mário de Andrade. Oder: Macunaíma, ein Wilhelm Meister in den Tropen?
}

[Tropical subjectivity and European cultural tradition: Macunaima, the hero with no character, by Mário de Andrade. Or: Macunaíma, a Wilhelm Meister from the tropics?]

\section{Horst Nitschack ${ }^{1}$}

\begin{abstract}
This article examines the many connections between the Brazilian novel Macunaíma, by Mário de Andrade, and the German cultural tradition, beginning with Hans Staden's narrative about "naked and ferocious savages, eaters of men," and ending with Koch-Grünberg's ethnological study From Roraima to Orinoco. Like the German coming-of-age story (Bildungsroman), Macunaíma should be read within the context of the formation and construction of national identity. But the European idea of formation is no longer a model for the construction of subjectivity in Brazil today. Macunaima's resistance to a modernity defined by rationality and to responsible action that confirms the reality of this modernity should not be read as a counter-model, but rather as a reaction of despair. Macunaima connects the coming-of-age story only to a critique of modernity (on the one hand, a critique with its own means and, on the other, an external critique), but also to the conviction that literary narratives can still be successful despite disillusionment toward the project of modernity.
\end{abstract}

Keywords: Cultural contact; coming-of-age story; subjectivity; cultural anthropophagy/ cannibalism; critique of modernity

Zusammenfassung: Der Artikel untersucht die vielfältigen Verschränkungen zwischen Mário de Andrades Roman Macunaima und der deutschen Kulturtradition, von Hans von Stadens Bericht über die „wilden nacketen grimmigen Menschenfresser Leuthen“ bis $\mathrm{zu}$ Koch-Grünberg ethnologischer Studie Vom Roraimo zum Orinoco. Dabei vertritt er die These, dass Andrades

\footnotetext{
${ }^{1}$ Professor da Universidad de Chile. Email: horst.nitschack@gmail.com

Dieser Artikel entstand im Zusammenhang des Forschungsprojektes Fondecyt $N^{\circ}$ 1120116: Rebeldes, malandros, delincuentes y locos: resistencias, transgresiones y expectativas utópicas en la literatura brasileña del siglo XX.
}

Pandaemonium, São Paulo, v. 16, n. 22, Dez/2013, p. 156-178 
Nitschack, H. - Macunaíma, ein Wilhelm Meister in den Tropen?

Roman, ähnlich wie der deutsche Bildungsroman, im Zusammenhang des Projekts der Herausbildung nationaler Identität zu lesen ist. Allerdings ist die europäische Bildungsidee kein Modell mehr für die Herausbildung von Subjektivität unter den brasilianischen Verhältnissen. Aber Macunaímas Widerstand gegen eine der Modernität verpflichtete Rationalität und ein die Realität dieser Modernität bestätigendes veranwortliches Handeln ist nicht als Gegenmodell zu lesen, sondern als eine Reaktion der Verzweiflung. Macunaíma verbindet mit dem Bildungsroman sowohl die Kritik der Moderne (einmal eine Kritik aus ihrer eigenen Mitte, im anderen Falle eine Kritik von aussen), wie auch die Überzeugung, dass (literarisches) Erzählen trotz aller Desillusion und aller Verzweiflung an dem Projekt der Moderne gelingen kann.

Schlüsselwörter: Kulturkontakte, Bildungsroman, Subjektivität, Kulturantropophagie, Modernitätskritik.

Resumo: O artigo investiga os diversos cruzamentos entre Macunaíma, romance de Mário de Andrade, e a tradição cultural alemã, partindo do relato de Hans Staden sobre os "selvagens nus e ferozes devoradores de homens", até o estudo etnológico De Roroima a Orinoco, de KochGrünberg. Sustenta-se a tese de que, à semelhança do romance de formação (Bildungsroman), o texto de Mário de Andrade tem de ser lido com referência ao projeto de desenvolvimento da identidade nacional. No entanto, a ideia europeia de formação já não é modelo para o desenvolvimento da subjetividade no contexto das relações brasileiras. Mas a recusa de Macunaíma a uma racionalidade comprometida com a modernidade e a uma ação responsável que confirma a realidade dessa modernidade não deve ser lida como contramodelo, senão como uma reação de desespero. Macunaíma vincula ao romance de formação tanto à crítica da modernidade - de um lado, uma crítica com seus próprios meios, de outro, uma crítica externa - quanto à convicção de que a narrativa (literária), apesar de toda a desilusão e todo o desespero no projeto da modernidade, pode ser bem-sucedida.

Palavras-chave: contato cultural, romance de formação, subjetividade, antropofagia cultural, crítica da modernidade.

Die Geschichte der Herausbildung der neuen Nationalstaaten seit dem 18. Jahrhundert ist auf vielfältige Weise verknüpft und verschränkt mit den Geschichten, die in ihnen erzählt werden und die diesen Prozess widerspiegeln, kommentieren, imaginär stimulieren und symbolische Referenzen bereitstellen. Sie erlauben ihren Lesern und fordern sie auf, sich mit den neu entstehenden und $\mathrm{zu}$ begründenden nationalen Gemeinschaften $\mathrm{zu}$ identifizieren oder warnen vor Irrwegen und einem möglichen Scheitern. Auf diese Weise leisten diese Erzählungen einen bedeutenden Beitrag zum Entstehen imaginärer nationaler Gemeinschaften. Im Vergleich ihrer eigenen Lebensläufe und Lebenserfahrungen mit 
Nitschack, H. - Macunaíma, ein Wilhelm Meister in den Tropen?

denen der Romanhelden, öffnet sich den Lesern ein Horizont, der ihren Erfahrungen und Erlebnissen eine Bedeutung gibt, die sie über ihre unmittelbare Lebenswelt hinausragen lässt und sie in eine nationale Gesamtheit einbindet.

Das erzählerische Medium, das hierfür geschaffen oder hierfür aktualisiert wird ist der Roman. Das gilt sowohl für die europäischen Nationen im 19. Jahrhundert, wie auch für die lateinamerikanischen (cf. SOMMER 1994), und das gilt - um uns unserem Thema mit einem Sprung zu nähern - für so unterschiedliche Nationen, wie dem in die europäischen Kulturtraditionen fest eingebundenen Deutschland und der neu entstehenden, erst seit 1822 endgültig aus der portugiesischen Kolonialherrschaft sich befreienden und damit unabhängigen tropischen Nation Brasilien.

Die ersten bedeutenden Manifestationen einer eigenständigen Romanerzählung in der Romantik, besonders die Romane José de Alencars, werden zwar ganz im tropischen Gewand auftreten und indianische Helden feiern, in ihrer Grundstruktur aber noch sehr europäisch geprägten Kulturmustern verbunden bleiben.

Der kulturelle „Befreiungsschlag“ findet in Brasilien mit dem Modernismus, so nennt sich die brasilianische Avantgarde de 20er Jahre des 20. Jahrhunderts, statt. Jetzt wird die brasilianische Kultur ihre Unabhängigkeit von der europäischen proklamieren, entschlossen in Malerei, Musik, Literatur von einer „Import-,, zu einer „Export-Nation“ zu werden, wie es einer der bedeutendsten Vertreter des brasilianischen Modernismus, Oswald de Andrade, provokativ in seinem Manifest „Pau Brasil““ (1924) verkündet hat. Wenige Jahre später, 1928, wird er sein zweites weitaus bekannteres Manifest veröffentlichen, das heute einer Kulturtheorie den Namen gibt (cf. JÁurgueI 2008; KECK/ Kording/ PROSCHASKA 1999): das antropophage Manifest. Antropophagie im metaphorischen Sinne: die Übernahme des Besten vom Gegner, ohne Berührungsangst, ohne die Befürchtung, damit die eigene Identität zu verlieren, sondern im Gegenteil, mit der Überzeugung, diese würde aus dieser Vereinnahmung des anderen gestärkt hervorgehen. Zeitgenosse und Mitstreiter Oswald de Andrades war Mário de Andrade. Er wird im gleichen Jahr, in dem Oswald de Andrade sein antropophages Manifest veröffentlicht seinen Roman Macunaíma, der Held ohne jeden Charakter (1997), vorlegen, den seine avantgardistischen Freunde, allen voran Oswald de Andrade, als den grossen antropophagen Roman der brasilianischen 
Nitschack, H. - Macunaíma, ein Wilhelm Meister in den Tropen?

Literatur feiern und der auch heute noch einer der bedeutendsten Romane im Kanon der nationalen Literatur ist.

Wenn wir nun auf den folgenden Seiten ein vielfältiges Beziehungsgeflecht zwischen diesem Roman und deutschen Kulturkulturtraditionen freilegen, so geschieht das zum einen mit der Absicht, auf komplexe Umwertungsprozesse und Uminterpretationsprozesse in diesem mehrfachen Hin- und Her hinzuweisen, zum anderen aber auch, um die These zu belegen, dass bei aller Unterschiedlichkeit von Herkunft und Lebensumständen zwischen diesem tropischen Helden ohne jeden Charakter und dem klassischen Helden des nördlichen Bildungsromans, Wilhelm Meister, mehr Ähnlichkeiten zu finden sind, als zu vermuten wäre.

Die Geschichte der folgenreichen Kulturkontakte zwischen der Welt Macunaímas und der Wilhelm Meisters beginnt bereits sehr früh, Mitte des 16. Jahrhunderts, etwa 50 Jahren nach der Entdeckung Brasiliens im Jahre 1500. Da verschlägt es den deutschen Landsknecht Hans von Staden ( geb. zwischen 1525 und 1529 in Homberg; $\uparrow$ unbekannt) in diese fernen Gegenden. Er gerät in die Gefangenschaft der Tupinambá, entkommt auf wunderbare Weise dem antropophagen Ritualen und veröffentlicht, wieder zurück in heimatlichen Gefilden, den europäischer „Bestseller“, die Warhaftige Historia und beschreibung eyner Landtschafft der wilden nacketen grimmigen Menschenfresser Leuthen in der Newenwelt America gelegen. ${ }^{2}$ Dieser „Testimonialtext“ ist einer der frühen Belege, wie europäische Mächte versuchen, diesen Erdteil in Besitz zu nehmen, ihn aber nicht nur ihrem Herrschaftsanspruch unterwerfen, sonden ihn gleichzeitig mit ihren Projektionen, Phantasien und Interpetationen $\mathrm{zu}$ besetzen versuchen. Nicht nur die zivilisatorischen Techniken, sondern auch Mythen, Fabelwesen und Schreckensphantasien werden über den Atlantik exportiert, von den einäugigen Riesen bis zu den männermordenden Amazonen und den menschenfressenden Kannibalen. So wird diese Neue Welt von Beginn an ambivalent besetzt und diese Ambivalenz wird sie bis in die Gegenwart hinein nicht

\footnotetext{
${ }^{2}$ Das Buch erscheint in der ersten Auflage 1557, erfährt noch im 16. Jahrhundert viele Neuauflagen, wird Vorlage für Raubdrucke und ins Französische übersetzt. Hans von Staden war ein Söldner und Landsknecht der zwei Reisen in die Neue Welt unternahm, in die Gegend des heutigen Rio de Janeiro. Auf der zweiten Reise wurde er von den Tupinamba gefangen genommen, die in jener Zeit für ihre anthropophagen Praktiken bekannt waren.
} 
Nitschack, H. - Macunaíma, ein Wilhelm Meister in den Tropen?

verlieren: als Raum der Hoffnung und als Raum der Schrecken. Wenn sie im 16. Jahrhundert bald Eldorado bald Hölle war, so wird sie im 20. Jahrhundert zum Ort politischer Utopien auf der einen und von Militärdiktaturen und Terrorismus auf der anderen Seite.

Diese Diskurse provozieren unterschiedliche Reaktionen auf lateinamerikanischer Seite. Das antropophage Manifest ist sicher eine der polemischsten, indem es die rhetorischen Strategie anwendet, eine Anschuldigung nicht zu widerlegen, sondern sie als Selbstbestätigung und Stärke zu interpretieren. Auf Stadens-Antropophagie-Vorwurf, der in der Folge vielfach wiederholt werden wird und der letztlich die Kultur- und Morallosigkeit der Tropen anprangert, antwortet fast 400 Jahre später Oswald de Andrade mit seinem antropophagen Manifest, dessen Titel bereits Programm ist: die Projektionen der europäischen Eroberer und Reisenden, die in der Nachfolge Stadens in jedem Indianer einen Kanibalen sehen wollen, werden aufgegriffen und polemisch zur Grundlage für eine Kulturstrategie gemacht, auf der die zukünftige Überlegenheit der tropischen Kulturen den europäischen gegenüber begründet ist: Nicht zur eigene Kultur durch Abgrenzung und Nabelschau finden, sondern nach dem Vorbild der Vorfahren den Gegner verspeisen: andere Kulturen vereinnahmen. So heißt der bekannteste Satz des Manifests, in dem das gesamte Programm kondensiert ist: „Tupi or not Tupi, that is the question.” (ANDRADE 1994: 47). Hier wird die ironische Identifikation mit den kannibalischen Vorfahren über ein Wortspiel zu dem die phonetischen Nähe von „to be“ und „Tupi“ einlädt, mit dem dramatischen und existentiellen Ausruf von Shakespeare's Hamlet: „To be or not to be“, „Sein oder nicht sein, das ist die Frage“, kurz geschlossen. Die Shakespeare'sche existentielle Seinsfrage wird provokativ uminterpretiert in: „Kanibale sein oder nicht sein, das ist hier die Frage.“. Damit aber proklamiert dieser Satz nicht nur Antropophagie als Kulturstrategie, sondern er praktiziert sie zugleich: Antropophagisch wird einer der großen Sätze der Weltliteratur transformiert, wird von seinem allgemeinen existentiellen Sinn befreit, wird zum Vehikel einer brasilianischen Forderung, der Forderung einer peripheren, kolonialisierten Kultur nach Anerkennung. Er wird zur Metapher für Befreiung und nutzt gleichzeitig in einer geradezu karnevalistischen Maskarade das Potential der Weltliteratur 
Nitschack, H. - Macunaíma, ein Wilhelm Meister in den Tropen?

für die eigenen Zwecke: hinter dem ent-stellten Satz der Weltliteratur ${ }^{3}$ verbirgt sich die Forderung nach eigener Identität, die sich aber nicht scheut, Elemente einer anderen Kultur, der kolonialisierenden Kultur, zum Zweck de Entkolonisierung einzusetzen. In einer entschlossenen Wende gegen die okzidentalen „Leitkulturen“ wird Antropophagie als universelles Prinzip gesetzt und damit polemisch argumentiert, dass diese Praktiken den tatsächlichen gesellschaftlichen Prozessen und Dynamiken wesentlich näher sind. Damit wird eine Überlegenheit der brasilianischen Kulturpraxis beansprucht, da sie - so die Überzeugung - die Unterdrückungen und Sublimationen der Kulturen der industrialierten, vor allem der europäischen Nationen aufhebt. Als theoretische Referenz wird Freuds Totem und Tabu, zitiert ${ }^{4}$, und gleichzeitig die antropophage Spekulation aus seinem „Das Unbehagen in der Kultur" vorausgenommen, das erst 1930, zwei Jahre nach dem Manifest erscheint: die rituelle Verspeisung (Verinnerlichung) des ermordeten Vaters durch die Söhne, deren Verdrängung, nach Freud, der Grund des Unbehagen in der Kultur ist.

Mário de Andrades Roman Macunaíma wird bei seinem Erscheinen von Oswald de Andrade als gelungenes Beispiel antropophager Strategie gefeiert werden. Allerdings handelt es sich dabei - wenn überhaupt - um eine ganz andere Art von (Kultur)Antropophagie als derjenigen, die Oswald proklamiert. Mário de Andrade betont das ausdrücklich in einem Brief an den bekannten Kritiker Tristão de Athayde (Alceu Amoroso Lima). ${ }^{5}$ Sein Roman ist weder ein Aufruf zur noch ein Beispiel für die ,antropophage Vereinnahmung avancierter europäische Kultur, Wissenschaft und Technologie ${ }^{6}$, wie es Oswald de Andrades antropophages Manifest proklamiert, wobei er auf Freud, Lévy-Bruhl, William James und Voronoff verweist, sondern im Falle von Mário de Andrade handelt es sich gewissermassen um eine ,Antropophagie zweiten Grades'. Sein ,antropophages Begehren` gilt den ethnologischen Berichten KOCH-GRÜNBERGS, Vom Roroima zum

\footnotetext{
${ }^{3}$ Das deutsche "entstellt" in seiner dreifachen Bedeutung: 1: deformieren, unkenntlich machen (sp. „desfigurar"); 2: im eigentlichen Wortsinn: frei stellen, ein Hindernis beseitigen; 3: das zur Erscheinung kommen lassen was vorher „verstellt“" war, die Aufhebung der „Verstellung“.

${ }^{4}$ Auf Freuds „Totem und Tabu“ (1913) wird im Anthropophagen Manifest explizit Bezug genommen und Freud wird als Referenz wiederholt erwähnt.

${ }^{5}$ Mário de Andrade an Manuel Bandeira zu seinem Verhältnis zur Antropophagie (ANDRADE 1997: 497)

${ }^{6}$ Das anthropophage Manifest ist sicher das erste Manifest, in dem das Fernsehen erwähnt wird, für das Anfang 1928 in Berlin gerade die ersten Übertragungsversuche gemacht wurden.
} 
Nitschack, H. - Macunaíma, ein Wilhelm Meister in den Tropen?

Orinoco (Berlin, 1917 und Stuttgart 1924), und dessen Aufzeichnungen der Mythen der Amazonas-Indianer, die, von Mário de Andrade teilweise aus dem Deutschen rückübersetzt, zu wichtigen Bestandteilen seines Romans werden. Was dieser deutsche Wissenschaftler in mühsamer und gefahrvoller Expedition durch unwegsame Gegenden mit wissenschaftlicher Exaktheit aufgezeichnet hat, ${ }^{7}$ die Mythen der Arekuna- und Taulipangindianer, wird von Mário de Andrade in eine Mischung aus Pikaro-, Bildungsroman und ethnologischer Satire verwandelt. Ein Buch - so die Selbstmytifizierung des Autors -, das darüber hinaus lustvoll in der Hängematte liegend innerhalb von sechs Ferientagen geschrieben worden sei (Cf. ANDRADE 1997: 497). Das scheint insofern zutreffend zu sein, als er mit dem aus Koch-Grünberg gewonnenen Material (dessen Herkunft er nur sehr selten erwähnt), innerhalb von wenigen Tagen die Konzeption der Roman-Rhapsodie entwirft. Dann vergeht allerdings noch das gesamte Jahr 1927, in dem er Kapitel des Manuskripts Freunden zu lesen gibt, andere um zusätzliches Material bittet, bevor zu Beginn 1928, fast gleichzeitig mit der Veröffentlichung des antropophagen Manifests Oswald de Andrades, der Text in den Druck geht. Auf die begeisterte Rezeption des Namensvetters, es handle sich um ein gelungenes Beispiel antropophagen Schreibens reagiert Mário de Andrade in dem schon erwähnten Brief an Tristão de Athayde (Alceu Amoroso Lima) geradezu ablehnend.

Dieser Roman, so haben wir zu Beginn argumentiert, hat für die brasilianische Literaturgeschichte eine ähnliche Funktion wie der klassische deutsche Bildungsroman, Wilhelm Meister, für die deutsche. Beide sind Romane, denen im literarischen nationalen Kanon eine privilegierte Stellung zukommt, jedesmal ist es die Geschichte eines Jugendlichen von der Geburt an, der in einer Reise (Odyssee) durch sein Land mit dessen unterschiedlichen Wirklichkeiten konfrontiert wird. Beide Helden müssen Prüfungen bestehen und erfahren Enttäuschungen und Leiden. Während Wilhelm allerdings am Ende eine Ehe mit Natalie schliesst, wird Macunaíma in einem blutigen Kampf mit der Sirene Uiara verstümmelt werden und als Stern in den Himmel aufsteigen. Aber das ist nur einer der zahlreichen signifikanten Unterschiede, die aber gleichzeitig nicht darüber hinweg

\footnotetext{
7 Koch-Grünberg war einer der ersten Ethnologen, der für seine ethnologischen Aufzeichnungen die avancierten technischen Mittel des Phonographen und des Films einsetzte. 
Nitschack, H. - Macunaíma, ein Wilhelm Meister in den Tropen?

täuschen dürfen, dass sich beide Fiktionen mit einer sehr vergleichbaren Situation konfrontieren: Die Möglichkeiten und Unmöglichkeiten eines Helden in der modernen Welt, genauer in der Nation und der Kultur, die er lernen muss, als seine eigene zu akzeptieren, seinen Ort zu finden. Dies ist die Grundfabel eines jeden Bildungsromans und das ist auch die Geschichte Macunaímas. Wenn Goethes Wilhelm Meister als erste grosse Verwirklichung des deutschen Bildungsromans gelten $\operatorname{kann}^{8}$, dann Mário de Andrades Macunaíma seine gelungene Transformation in die tropische Welt Brasiliens angesichts der Herausforderung der Herausbildung einer - ähnlich wie im Falle Wilhelm Meisters unabhängen Nationalliteratur.

Wilhelm Meister und Macunaíma vergleichen zu wollen, lässt sich formal rechtfertigen, wenn wir daran denken, welche Rolle den Figuren - nicht nur den Romanen - im Imaginären der deutschen einerseits und der brasilianischen Bildungstradition andererseits zukommt. Auf den ersten Blick ist allerdings bei dieser Migration vom deutschen Wilhelm Meister nur sehr wenig übrig geblieben. Das kann auch keinesfalls verwundern, wenn wir bedenken, unter welchen grundsätzlich verschiedenen Bedingungen Subjektivität in der tropischen Welt möglich wird. ${ }^{9}$

Dass die tropische Welt ein problematischer Ort für das nördliche Subjekt ist, hat bereits J. W. Goethe seine Ottilie in den Wahlverwandschaften ahnen lassen, wenn sie ihren Wunsch, ,Humboldten erzählen zu hören“ selbst kommentiert: „Es wandelt niemand ungestraft unter Palmen, und die Gesinnungen ändern sich gewiss in einem Lande, wo Elefanten und Tiger zu Hause sind.“ (GoETHE 1981: 416). Der Gesinnungswandel, der gleichzeitig einen Wandel von Wertvorstellungen und Urteilskriterien bedeutet, muss nach Ottilie als Strafe interpretiert werden. Nicht weniger drastisch hat ein ganz anderer Reisender, Ernst Jünger, die Gefahr der tropischen Welt für den westlichen Reisenden und seine Subjektivität beschrieben:

\footnotetext{
${ }^{8}$ Friedrich Schlegel über Goethe's Wilhelm Meister Lehrjahre: »Die Französische Revolution, Fichtes Wissenschaftslehre, und Goethes Meister sind die größten Tendenzen des Zeitalters." Athenäum, Fragment 216: KFSA 2, S. 198 (Nr. 216).

${ }^{9}$ Im deutschsprachigen Raum gab es eine Adaptierung des Wilhelm Meister in den 70er Jahren, dessen Protagonist ebenfalls nur sehr ferne Ähnlichkeiten mit seinem Vorbild aufweist, obwohl er seine Reise durch Deutschland selbst macht: Wim Wenders Langsame Bewegung nach dem Drehbuch von Peter Handke.
} 
Nitschack, H. - Macunaíma, ein Wilhelm Meister in den Tropen?

Während dieses Vorübergleitens [bei der Einfahrt in das Amazonasdelta] malte ich mir ein Dasein aus, das zwischen den Armen und Inseln dieses ungeheuerlichen Deltas zu führen wäre, sei es, dass man als Jäger oder Fischer, sei es als Gärtner oder einfach als Beobachter des Stromes und seiner gewaltigen Fülle lebendiger Vorgänge sich ansiedele. Doch würde man es wohl nicht lange treiben; das Wachstum ist zu ungeheuer, als dass man ihm auf die Dauer standhielte. Man würde im reinen Sauerstoff verbrennen und müsste mit einem frühen Tode, mit geistiger und körperlicher Verheerung rechnen, mit einem Schicksal, ähnlich dem Rimbauds. (JÜNGER 1949: 17).

Den Aufzeichnungen des bedeutendsten europäischen „Reisenden“ in den amerikanischen Tropen, Alexander von Humboldts Reise in die Aequinoctial-Gegenden des neuen Continents, können wir sehr gut entnehmen, wie er sich gegen die Entropie tropischer Auflösung geschützt hat: durch das unablässige Vermessen dieser Welt, durch den Versuch ihrer möglichst lückenlosen Registrierung, durch ihre genaue Einschreibung in die Koordinaten objektiver Naturwissenschaft. Alexander Humboldts Reisebeschreibung ist ein eindrucksvoller Beleg dafür, dass die Eroberung der fremden Länder und die Unterwerfung der Natur dem modernen Subjekt nicht ohne eine gleichzeitige konsequente Selbstkontrolle und die Unterwerfung der eignen Natur möglich ist. Die radikale Selbstbeherrschung ist der Preis, den dieses Subjekt für die Beherrschung der Welt bezahlt. Die Überzeugung von der Vermessbarkeit der Welt, zusammen mit der Überzeugung, dass nur was gemessen werden kann überhaupt Bedeutung hat, ist Voraussetzung für die Unzahl der Naturforscher, die sich ab dem 18. Jahrhundert von Europa aus über die Welt verbreiten, um Gesteine, Pflanzen, Tiere und später auch die Menschen den in den europäischen Akademien und von europäischen Gelehrten entwickelten und ausgedachten Ordnungsprinzipien zu unterwerfen. Diese wissenschaftliche Kolonisierung geht einher mit der wirtschaftlichen und politischen und ist oftmals sogar ihre Voraussetzung. Auf lateinamerikanischer, in unserem Fall ganz konkret auf brasilianischer Seite wird dieser Rationalität, dem zweckrationalen Handeln und dem Effizienzdenken der nördlichen Industrieländer, die als historische Herausforderung am Horizont stehen, von vielen Intellektuellen und Künstlern mit Skepsis und Kritik mit begegnet werden, eine Haltung die allerdings auch in Europa selbst nicht selten ist. Die Entdeckung exotischer Welten durch europäische Künstler vor und nach der Wende vom 19. zum 20. Jahrhundert, wie im Falle von Gauguin oder etwas später von Carl EINSTEIN (1915), ist nicht nur Zivilisationsflucht, sondern auch eine 
Nitschack, H. - Macunaíma, ein Wilhelm Meister in den Tropen?

Reaktion auf den vehementen Modernisierungsprozess. Dem entspricht in Brasilien die Entdeckung der ,eigenen“ indianischen Welten (deren kulturelle Eigenständigkeit erst heute langsam anerkannt wird, nachdem der Kontakt zur indianischen Bevölkerung vor allem durch die Enteignung und Nutzung der von ihr besiedelten Räume gekennzeichnet war), der allerdings nicht mit einer radikalen Kritik der Modernisierung einhergeht, sondern eher durch Ambivalenz bestimmt ist. Der erste Weltkrieg und seine Folgen werden von zahlreichen lateinamerikanischen Intellektuellen durchaus als „Untergang des Abendlandes“ interpretiert - und das gleichnamige Spenglersche Werk ${ }^{10}$ wird, entgegen seiner tatsächlichen Argumentation, dafür als Beleg angeführt - aber das hatte keineswegs eine radikale Technikfeindlichkeit zu Folge, sondern vielmehr den Wunsch nach einer utopischen Verschmelzung von Technik und „primitiven“ Kulturen (ANDRADE 1990). Oswald de Andrade wird später als zukünftige Synthese den ,natürlichen technisierten Menschen" fordern, den "homem natural tecnizado" (id.: 13) und damit wieder seine Idee vom technisierten Barbaren aufgreifen, "o bárbaro tecnizado" (id.: 48), die bereits im Antropophagen Manifest zitiert ist und bei der er sich (in einem Missverständnis, wie es auch in der Rezeption Spenglers bereits der Fall war) auf den Reisenden und Philosophen Hermann KeYSERLING (1919) beruft.

Mário de Andrades Macunaíma, so werden wir im Folgenden argumentieren, ist zwar auch eine avantgardistische Adaptierung indianischer Mythen auf dem Umweg über ihre Übersetzung ins Deutsche, ${ }^{11}$ und ihre Rückübersetzung ins brasilianische Portugiesische, mehr noch aber ist der Roman die sarkastische Auseinandersetzung mit einer Vernunft- und Moralkritik, die sich auf das Natürliche und den „guten Wilden“ (bon sauvage) zu berufen sucht, wie es im Modernismus ${ }^{12}$ Mode geworden ist (cf. NITSCHACK 2001: 108-118). Mário de Andrade als Autor „leidet“, wie er schreibt, mit seinem Helden Macunaíma, aber das heisst nicht, dass er sich - wie es die Kritik vielfach behauptet - mit

\footnotetext{
${ }^{10}$ „Der Titel, seit 1912 feststehend, bezeichnet in strengster Wortbedeutung und im Hinblick auf den Untergang der Antike eine welthistorische Phase vom Umfang mehrerer Jahrhunderte, in deren Anfang wir gegenwärtig stehen." SPENGLER (1912: Bd. 1, p. X).

${ }^{11}$ Die Aufzeichnungen der Mythen finden sich im 2. Band, die 1924 in Stuttgart veröffentlich werden. Der 1. Band ist die Reisebeschreibung, veröffentlicht 1917 in Berlin.

${ }^{12}$ Modernismo: Bezeichnung für die Avantgarde-Bewegung zu Beginn des 20. Jahrhunderts in Brasilien und Portugal.
} 
Nitschack, H. - Macunaíma, ein Wilhelm Meister in den Tropen?

dessen A-Moralität idenfiziert. Hier wiederholt sich die Desillusion des Bildungsromans, des deutschen zumal, aber auch des französischen ${ }^{13}$, auf ganz neue Weise. Das Bildungsmodell hat sich in der europäischen Tradition von Beginn an als sehr widersprüchlich erwiesen: dieses ideale Subjekt, zu dem das Individuum in seinem Bildungsprozess sich verwandeln soll und dessen Verwirklichung von ihm erwartet wird, war unter diesen historischen, sozialen und politischen Bedingungen gar nicht realisierbar. Das Individuum geriet in eine paradoxe Situation: die Gesellschaft forderte von ihm auf der einen Seite, sich zu einem verantwortungsvollen Subjekt zu bilden und gleichzeitig machte sie ihm das aufgrund ihrer konkreten Verhältnisse unmöglich. So wird der Bildungsroman bereits von Anfang an ein Desillusionsroman, ein Roman der seinen Protagonisten lehrt, dass diese Ideale keinesfalls im wirklichen Leben ihre Realisierung finden können, sondern allenfalls in der Innerlichkeit, getragen von der Bereitschaft zur Entsagung. ${ }^{14}$ Wo es diese Bereitschaft auf Verzicht und die Einsicht in die Notwendigkeit, sich den Gesetzen zu unterwerfen nicht gibt, droht das Scheitern, wie im Falle von Stendhals Lucien. Wilhelm Meisters Ende ist zwar etwas versöhnlicher, er wird am Ende eine Ehe mit Natalie eingehen, um sich gleich darauf alleine mit seinem Sohn Felix (ein Kind aus seinem Abenteuer mit Marianne, seiner ersten Leidenschaft) nach Italien auf die Reise zu begeben, aber gleichzeitig ist dieses Ende durch Mignons Tod und des Harfners Selbstmord gezeichnet.

Auch Macunaímas Ende ist das eines gewaltsamen Scheiterns. Er scheitert als „Held“, der aus den Tiefen des Amazonasurwalds auftauchte und als satirischer Kritiker einer ihm fremden Rationalität und einer abstrakten Logik, seine abenteuerliche Odyssee durch Brasilien bis in das mit den letzten Errungenschaften technischer Modernität ausgestattete São Paulo unternimmt. Der Lieblingssatz dieses ,Helden ohne jeden Charakter", "Que preguiça“" -,,welche Faulheit" -, der sich wie ein Leitmotiv durch den Roman zieht, ist sowohl die Kritik des protestantischen Arbeitsethos der erfolgreichen

13 Bei Stendhal (Le Rouge et le Noire), BAlzaC (Les Illusions perdues) und FlaUBERT (Education Sentimentale) gleichermassen.

14 „Menschentypus und Handlungsstruktur sind also hier von der formalen Notwendigkeit bedingt, dass die Versöhnung von Innerlichkeit und Welt problematisch aber möglich ist; dass sie nach schweren Kämpfen und Irrfahrten gesucht werden muss, aber doch gefunden weden kann.“ (LUKÁCS 1920: 141)

Pandaemonium, São Paulo, v. 16, n. 22, Dez/2013, p. 156-178 
Nitschack, H. - Macunaíma, ein Wilhelm Meister in den Tropen?

Industriegesellschaften, wie auch die eines romantisierenden Indianerbildes und einer gelobten primitiven Welt, wie sie von der Romantik an bis in den Modernismus hinein immer wieder in der brasilianischen Literatur gefeiert werden. Macunaíma hat nichts von einem „homem natural tecnizado“, einem ,technisierten Naturmenschen“, Oswald de Andrades. Von Koch-Grünberg hat Mário de Andrade die einzelnen Episoden übernommen (cf. CAVAlCANTI PROENÇA 1973), aber die Aktualisierung dieses mythischen Materials und die literarische Gesamtkomposition ist fraglos seine Leistung. Sie beginnt nach dem Modell mytischer Berichte mit Zeugung, Geburt und Kindheit, deren Außergewöhnlichkeit die ersten Zeichen der Bestimmung des Helden sind. Gleichzeitig aber setzt hier sofort die Satire auf die Idealisierung der indianischen Kultur ein, die den brasilianische Indianismus der Romantik kennzeichnet (bei Gonçalves de Magalhães, José de Alencar und Gonçalves Dias auf jeweils sehr unterschiedliche Weise), der seinerseits die nördlichen Idealisierungen eines Chateaubriand (Atala und René) und eines Fenimore Cooper (The Last of The Mohicans) $\mathrm{zu}$ einem edlen brasilianischen Indianer uminterpretiert hatte. Macunaíma erhält zu Beginn von Ci, der Mutter des Urwaldes, einen im Grunde wertlosen Stein, die Muiraquitã, zum Geschenk. Dieser wird ihm geraubt und der Held macht sich quer durch das weite Brasilien auf die Suche nach ihm. Dabei gelingt es ihm zwar seine Muiraquitã wieder zu gewinnen, aber nur, um sie dann am Ende bei einem leidenschaftlichen Liebesabenteuer mit der Sirene Uiara erneut $\mathrm{zu}$ verlieren. In dem Liebeszweikampf wird ihm obendrein auch noch sein rechtes Bein abgebissen. So verabschiedet sich der Held aus dem Leben und steigt als Stern ins Bild des Großen Bären auf.

Macuaíma, als ,Held ohne jeden Charakter', ist vollständig amoralisch, egoistisch, faul, er vereinigt alle negativen Charakterzüge, die Mário de Andrade bei seinen Landsleuten $\mathrm{zu}$ finden meint, ohne irgendeine seiner positiven in Erscheinung treten $\mathrm{zu}$ lassen. ${ }^{15}$ Mário de Andrade ist fasziniert von der Negativität Macunaímas, der mythischen Welt dieses Helden und ihrer Irrationalität. Ihre Vermischung von Traum und Wirklichkeit, ihre triebhafte Sexualität, ihre Bereitschaft zu den unterschiedlichsten Formen von Gewalt

\footnotetext{
${ }^{15}$ Siehe Brief an Manuel Bandeira, 7. November 1927: „... do qual eu procurava tirar todos os valores nacionais" [" $\ldots$ dem ich alle nationalen Werte zu nehmen suchte"] (ANDRADE 1997: 495).

Pandaemonium, São Paulo, v. 16, n. 22, Dez/2013, p. 156-178
} 
Nitschack, H. - Macunaíma, ein Wilhelm Meister in den Tropen?

und ihre Verantwortungslosigkeit wird jedoch in keinem Moment als Beispiel eines modernitätskritischen Verhaltens vorgestellt.

Wenn auch eine ganze Reihe von Kritikern das subversive oder das kontrahegemoniale Potential der „malandragem“16 Macunaímas positiv hervorheben, so tritt doch eine solche Aufwertung in Widerspruch zu den Selbstkommentaren Mário de Andrades zu seinem Helden. In seiner Korrespondenz unterscheidet er von Anfang an sehr klar zwischen seiner poetischen Arbeit- und in der Hinsicht scheint ihm die ,Rhapsodie‘, wie Mário de Andrade selbst den Roman nennt, von Anfang an geglückt - und der (A-) Moralität seines Helden, die er immer als problematisch ansieht. Zu Beginn sieht er in ihm vor allem die komischen Züge und die Satire auf brasilianische Zustände, in den 40er Jahren wird er sich allerdings dann mit deutlicher Kritik an seinem eigenen Text äußern. Die „malandragem“ seines Helden, seine Unabhängigkeit von moralischen Urteilen, seine Infragestellung aller Regeln, die die Kritik später als positive Qualitäten hervorhebt, sind für den Autor durch und durch negativ konnotiert. In einem Brief an den jungen Autor Fernando Sabino von 1942 - das Jahr in dem er mehrfach mit seinem Helden haderte finden wir folgende Sätze:

O que eu tenho sofrido com Macunaíma, principalmente com ele, você nem pode imaginar... E no entanto, si escrito em pleno estado de possessão (a primeira redação foi feita inteirinha em seis dias), em que eu não sofria nada no ímpeto sublime da criação, mas também nem podia pôr consciência na sublimidade em que estava pela extensão mesma desta sublimidade que me obnubilava qualquer estado de consciência analítica, o que posso lhe jurar é que Macunaíma foi detestavelmente doloroso pra mim. Nos momentos mais anedóticos, mais engraçados do entrecho, eu não deixava de sofrer pelo meu herói, sofrer a falta de organização moral dele (do brasileiro, que ele satiriza), de reprovar o que ele estava fazendo contra a minha vontade. (ANDRADE 1997: 514) ${ }^{17}$

\footnotetext{
${ }^{16}$ Der ,malandro", dessen (Un)-Taten die "malandragem" ist, ist eine brasilianische Version des Pikaros. Ihn charakterisieren die kleinen, meist liebenswürdigen Übertretungen des Gesetzes, die vor allem dessen Absurditäten und Ungerechtigkeiten deutlich werden lassen. Die vom „malandro“ Geschädigten sind meist selbst daran schuld und finden beim Leser weder Mitleid noch Verständnis. Dieser ist immer auf Seiten des ,malandros'.

17 „Sie können sich nicht vorstellen, wie ich an Macunaíma - an ihm vor allem - gelitten habe. ...Wenn also [der Text] in einem Zustand vollständiger Besessenheit geschrieben wurde (die gesamte erste Niederschrift fand innerhalb von sechs Tagen statt), unter dem ich, da ich unter dem erhabenen Eindruck des Schaffens stand nicht litt, bei dem ich aber diese Erhabenheit auch nicht mit Bewusstsein wahrnehmen konnte, da ich
} 
Nitschack, H. - Macunaíma, ein Wilhelm Meister in den Tropen?

Auch in einem Brief an die Dichterin Henriqueta Lisboa, beklagt er die Unmoral (,desmoralidade“) des Helden (id.: 515), bzw. seine Amoralität (,imoralidade“). Aber er insistiert gleichzeitig darauf, dass die von ihm dargestellte Immoralität, künstlerisch verarbeitet war:

[...] e si os deu a público foi porque sentí a necessidade essencial deles. Necessidade minha, pessoal e necessidade humana, dos outros. Aqui você ainda poderá concordar comigo no caso do Macunaíma que é uma sátira, uma crítica, e cujo herói eu castigo e advirto no final, fazendo ele ir viver, por incapaz de uma vida fecunda, o brilho inútil das estrelas. (ANDRADE 1997: 515)

Die entschiedenste Selbstkritik lesen wir dann in einem Brief an Álvaro Lins:

Mas a verdade é que eu fracassei. Si o livro é todo ele uma sátira, um não conformismo revoltado sobre o que é, o que eu sinto e vejo que é o brasileiro, o aspecto 'gozado' prevaleceu. É certo que eu fracassei. Porque não me satisfaz botar a culpa nos brasileiros, a culpa tem de ser minha, porque quem escreveu o livro fui eu. Veja no livrinho a introdução com que me saudaram! Para esses moços, como pra os modernistas da minha geração, o Macunaíma é a 'projeção lírica do sentimento brasileiro, é a alma do Brasil virgem e desconhecida!' Que virgem nada! Que desconhecida nada! Virgem, meu Deus! Será muito mais um cão nazista! Eu fracassei. (id.) ${ }^{18}$

Die kritische Dimension seines Buches, beklagt Mário de Andrade, wurde nur von wenigen erkannt, auch nicht von denen, die darüber nachgedacht haben. Denn es waren entweder: "uns porque-me ufano-do-meu-país que recusaram a sátira e continuaram muito satisfeitos

durch ihr Ausmass ganz gefangen war, indem sie mir jegliches analytische Bewusstseins trübte, so kann ich Ihnen doch beteuern, dass Macunaíma fürchterlich schmerzlich für mich war. Selbst in den höchst anekdotischen Momenten, bei den witzigsten Passagen, hörte ich nicht auf, für meinen Helden zu leiden, an dem Fehlen seiner Disposition zur Moral zu leiden (die die des Brasilianers war, den er satirisch verkörperte), zu verurteilen, was er gegen meinen Willen tat.“

18 “Aber die Wahrheit ist, ich bin gescheitert. Wenn das ganze Buch eine Satire ist, ein revoltierender NichtKonformismus, darüber was der Brasilianer ist, wie ich es fühle und sehe, so hatte der genüssliche Aspekt doch den Vorrang. Es ist wahr, ich bin gescheitet. Denn es befriedigt mich nicht, den Brasilianern die Schuld zuzuschieben, die Schuld muss bei mir liegen, denn ich war es, der das Buch geschrieben hat. Schau Dir in dem Büchlein das Vorwort an, mit dem sie mich feierten! Für diese Dummköpfe, wie für die Modernisten meiner Generation, ist Macunaíma, die lyrische Projektion des brasilianischen Gefühls, ist er die jungfräuliche und unbekannte brasilianische Seele!' Nichts von jungfräulich! Nichts von unbekannt. Jungfrau, mein Gott! Er ist viel mehr ein Nazistischer Teufel. Ich bin gescheitert.“

Pandaemonium, São Paulo, v. 16, n. 22, Dez/2013, p. 156-178 
Nitschack, H. - Macunaíma, ein Wilhelm Meister in den Tropen?

da vida, ou foram os que só retiraram do livro um reforço consciente de seu amoralismo ... nacional." (id.: 514) ${ }^{19}$

Macunaíma ist nach diesen Zitaten aus Mário de Andrades Korrespondenz eine Satire auf eine intellektuelle Haltung, für die Amoralität und Irrationalität eine geeignete Gegenreaktion auf die Modernisierung des Landes durch eine ihm fremde Rationalität und Leistungsmoral ist. Das Besondere an Mário de Andrades Position ist, dass er weder eine brasilianische Wirklichkeit, die sich einem zweckrationalen Modernisierungprozess unterwirft, noch eine Kritik daran, die eine Verherrlichung des Mythischen und Irrationalen feiert, akzeptieren will. Weder sind für ihn die verschiedenen Kultur- und Technikadaptationen, die Brasilien von den nördlichen industrialisierten Nationen übernimmt eine Lösung, noch die Oswald'sche Utopie eines „technisierten Barbaren“, oder die Verherrlichung von mythischer Irrationalität, Gewalt und sexuellem Begehren im Stile Macunaímas. Denn wie in jedem literarischen Text darf auch in diesem Falle der Leser die Amoral des Helden nicht mit den Überzeugungen des Autors verwechseln. Mário de Andrade selbst macht klar und betont, dass die Rhapsodie nicht unmoralisch ist, sondern dass er ,auch die Inmoralität benutzt, nicht meine, sondern die meines Helden, um die moralische Unzulänglichkeit des brasilianischen Menschen zu charakterisieren.“ (id.: 515)

Macunaíma wird durch Brasilien getrieben, aber seine Suche nach dem verlorenen Stein ist nicht die Suche nach der erlösenden Kraft des Grals. Seine Odyssee endet für ihn nicht in einem Ithaka - auch wenn er am Ende wieder in den Amazonas-Urwald zurückkehrt, allerdings nur, um von dort, nach dem erneuten Verlust des Steines, verstümmelt in den Himmel aufzusteigen und zu einem Stern des Grossen Bären zu werden. Die Komik seiner Abenteuer ruft beim Helden wie beim Leser eher ein Lachen der Verzweiflung als eines der Befreiung hervor. Seine triebhafte sexuelle Besessenheit ist mehr ein selbstzerstörerischer Genuss, als die Voraussetzung für eine Lust an der gegenseitigen Anerkennung in einer geglückten Liebesbeziehung. Macunaíma ist noch auf der Suche, einer bei allem Lachen eher verzweifelten Suche, nach dem Weg, der in eine

19, „[...] solche für die Brasilien das Größte ist und die die Satire nicht wahrhaben wollten und die sehr zufrieden mit ihrem Leben weiter machten, oder solche, die aus dem Buch nur eine bewusste Bestätigung ihres - nationalen - Amoralismus herauslasen.“

Pandaemonium, São Paulo, v. 16, n. 22, Dez/2013, p. 156-178 
Nitschack, H. - Macunaíma, ein Wilhelm Meister in den Tropen?

zukünftige Kultur Brasiliens führen könnte. Ein ,Held ohne jeden Charakter` zu sein ist kein Glück, sondern ein Verhängnis. Macunaíma ist von Mário de Andrade mit einer tiefen Sehnsucht nach einer wirklichen und authentischen brasilianischen Kultur geschrieben, einer Kultur, in der die regionalen Widersprüche, die historischen Ungleichzeitigkeiten des Landes, sozialen Strukturen, die ein solidarisches Handeln und eine Anerkennung des Anderen unmöglich machen, überwunden werden. Wenn wir jetzt am Ende nochmals Macunaíma und Wilhelm Meister vergleichen, so ist bei allen historischen und kulturellen Unterschieden Macunaíma sicher das radikalere und verzweifeltere Buch. Die Hoffnung auf die Kraft der Bildungsidee, auf eine tiefe Übereinstimmung von antropologischer Disposition zur Bildung und von Bildungsinhalten, die - so im Goetheschen Fall eineseits aus der antiken Vergangenheit, vermittelt über Italien an uns ,,vererbt“ sind und nur neu „erworben“ werden müssen, andereseits aber auch in der europäischen Moderne bereits hervortraten (Shakespeare: in diesem Fall ein existentielles „To be or not to be“ Hamlets und nicht das „Tupi or not tupi“ Oswald de Andrades!!) kennt Macunaíma nicht. Der vielzitierte Lukács’sche Satz zum Helden des Bildungsroman, „,begonnen ist der Weg, vollendet die Reise“ (LuKÁCS 1920: 66), gilt nicht für ihn. Seine Reise ist beendet, ohne dass er einen Weg gefunden hätte. Sein Ende ähnelt, vor allem wenn wir es vor dem Hintergrund der von uns zitierten Kommentare des Autors selbst lesen, viel mehr Hyperions vorletzten Brief, den Hölderlin ganz der Verzweiflung seines Helden widmet, wieder „unter den Deutschen“ leben zu müssen. Der deutsche wie der brasilianische Fall, die beide durch mehr als hundert Jahre getrennt sind und auf sehr unterschiedliche kulturelle Kontexte antworten, stehen vor dem gleichen Dilemma: Der Wunsch nach der Herausbildung einer Nationalkultur bei der gleichzeitigen Einsicht in die Unmöglichkeit dieses Projekts, da Ideal und Wirklichkeit sich unversöhnlich gegenüber stehen. Dass das Entstehen einer nationale Kultur notwendig ist, diese sich aber nur in einem internationalen Kontext herausbilden kann scheint Mário de Andrade auf ganz ähnliche Weise zu spüren wie die Goethezeit: Wilhelm Meister macht sich am Ende auf den Weg nach Italien, Mário de Andrade hat die Hoffnung, sein Macunaíma könnte durch eine Übersetzung in eine andere Sprache gewinnen. So lesen wir in einem der Briefe an Manuel Bandeira 
Nitschack, H. - Macunaíma, ein Wilhelm Meister in den Tropen?

(12.12.1930), in dem er die ersten Versuche der Übersetzung seiner Rapsodie ins Englische kommentiert:

[...] talvez o Macunaíma ganhe em inglês porque muito secretamente o que me parece é que a sátira, além de dirigível ao brasileiro em geral, de que mostra alguns aspectos característicos, escondendo os aspectos bons sistematicamente, o certo é que sempre me pareceu também uma sátira mais universal ao homem contemporáneo, principalmente sob o ponto-de-vista desta sem-vontade itinerante, destas noções morais criadas no momento de as realizar, que sinto e vejo tanto no homem de agora. (ANDRADE 1997: 509-510) ${ }^{20}$

Es handelt sich bei Macunaíma also sehr wohl auch um eine Kritik der Modernität, die mit ihren radikalen Modernisierungsverfahren - um nochmals LUKÁCS (1920: 24) zu Wort kommen zu lassen - zur Ursache einer ,transzendentalen Obdachlosigkeit“ wird. Hier berührt sich Mário de Andrades Macunaíma dann wieder mit Beobachtungen des Forschungsreisenden Koch-Grünberg, der in den abgelegendsten Siedlungen des Amazonas die zerstörende Kraft der westlichen Zivilisation und die Folgen dieser Modernisierung zu sehen und zu spüren bekommt. Überall bewirkt sie Vermischungen und Hybridisierungen, die die lokalen Lebensformen und Traditionen zerstören und an ihre Stelle Neues treten lassen, das mit denen, die es bald spielerisch, bald aus bequemer Anpassung, bald aus Verzweiflung übernehmen nichts zu tun hat. Dieses Neue tatsächlich sich anzueignen, das

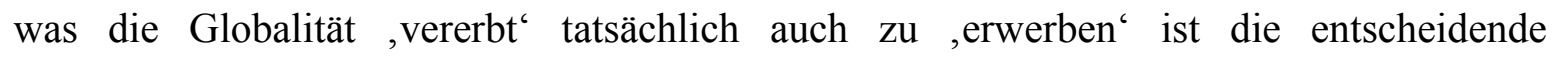
Herausforderung. Der von der (Kultur)-Antropophagie angepriesene „Verdauungsprozeß“ ist auf keinen Fall ein natürlicher, sondern er erfordert ein großes Maß an kultureller Arbeit. Das hat Oswald de Andrade in seinem Manifest unterschlagen und Mário de Andrade überspielt es in der Mythifizierung seiner Niederschrift des Romans. In der tragischen Aussichtslosigkeit seines Helden, einen Weg zu finden wird es aber sehr wohl zum Thema. Die spielerische Übernahme Elemente anderer Kulturen führen zu komischem Patchwork, das dem distanzierten Betrachter - selbst wenn er professioneller Ethnologe ist, wie

\footnotetext{
20 “... vielleicht wird Macunaíma im Englischen gewinnen, denn ganz heimlich erscheint mir, die Satire ist nicht nur an den Brasilianer gerichtet, von dem er einige charakteristische Aspekte zeigt, wobei seine guten Seiten ganz systematisch versteckt werden, wahr ist, dass sie mir auch immer als eine mehr universelle Satire über den gegenwärtigen Menschen erschien, vor allem unter dem Gesichtspunkt dieser ziellosen Willenlosigkeit, dieser moralischen Prinzipien, die im Moment ihrer Anwendung formuliert werden, wie ich es beim heutigen Menschen fühle und sehe."
}

Pandaemonium, São Paulo, v. 16, n. 22, Dez/2013, p. 156-178 
Nitschack, H. - Macunaíma, ein Wilhelm Meister in den Tropen?

Theodor Koch-Grünberg (1917: 80), mit viel Sympathie und Interesse für die indianischen Kulturen, - als hilflose karnevalistische Inszenierung erscheinen muss:

Am 7. September beginnen die Tänze in der bekannten Weise. Pitás Festtracht verdient beschrieben zu werden. Mit einigen Sachen, die ich im schenkte, hat er sich wunderbar zurechtgemacht. An einer alten Offiziersschärpe, die seine Lenden umgürtet, hängt ein Infanterie-Seitengewehr. Auf seine graue Zwillichjacke hat ihm seine Frau stolze Pionierepauletten genäht. Auf dem Kopf trägt er eine Radfahrermütze. Die Marke, die das Futter trug, hat er vorn an die Mütze geklebt, und so prangt über seinem ewig lachenden, schlauen Gesicht die bezeichnende Inschrift ,Tip-top“!

Bei aller Bewunderung der bricolage, des Fragmentarischen, des Hybriden ist zu fragen, ob es sich dabei nicht nur um Übergangsphasen handel kann, deren Kreativität hoch zu schätzen ist, die aber immer auch den Wunsch nach neuen Ordnungen in sich tragen, die die durch den Kontakt mit Modernisierungsprozessen provozierten Desintegrationen durch die Rückkehr zum Archaischen zu kompensieren suchen. In diesem Sinne ist der Amoralismus Macunaímas ähnlich archaisch wie die Rituale in den neuen tribalen Gruppen der Vorstädte. Nur stand Mário de Andrade der Rückkehr zu mythischen Archaismen wesentlich kritischer gegenüber als Oswald. Für Mário de Andrade war die Antropophagie des Riesen Piaimã in Macunaíma Teil seiner Satire.

Macunaíma als einen (gescheiterten) Bildungsroman $\mathrm{zu}$ lesen, eine Wahlverwandtschaft zwischen Wilhelm Meister und dem „Helden ohne jeden Charakter“ auszumachen, bedeutet nicht eine literarische Abhängigkeit des brasilianischen Textes von dem deutschen Klassiker zu behaupten, obwohl es, wie wir gesehen haben, vielfältige Bezüge zur europäischen und besonders auch zur deutschen Kultur gibt, sondern es bedeutet freizulegen, dass unter vergleichbaren historischen Herausforderungen, wie der Herausbildung eines Nationalstaates und der Suche nach einer subjektiven Moralität, die das Individuum gleichzeitig zu einem verantwortungsbewussten „citizen“ (cidadão) werden lässt, vergleichbare, wenn auch in sich wieder sehr unterschiedliche Antworten gegeben werden. Im Gegensatz zu Wilhelm Meister steht bei Mário de Andrade kein Bildungsideal im Hintergrund und die Hoffnung, dieses liesse sich im Werdegang des Helden verwirklichen. Hier steht Macunaíma dem Pikaro-Roman viel näher als dem Bildungsroman. Bei allen Metamorphosen, die der Held vollzieht bleibt er bis zum letzten 
Nitschack, H. - Macunaíma, ein Wilhelm Meister in den Tropen?

Abenteuer ein „Held ohne jeden Charakter“. Aber bereits im Falle des paradigmatischen Bildungsroman Wilhelm Meister haben zeitgenössische Kritiker das Scheitern des Bildungsideals bemerkt. Der berühmte Kritiker NICOLAI (1987: 89) spricht, vom ,,armen Meister“, „der in seinen Lehrjahren nichts gelernt hat, als sich von jedem Geschöpfe regieren zu lassen, das er antraf $[\ldots]^{“ 6}$.

Die Aporien des Bildungsideals, als ein Ideal das gesellschaftlich gefordert, dessen Realisierung aber gleichzeitig unmöglich gemacht wird, von denen alle Bildungsromane ein Zeugnis ablegen, wiederholen sich in der postkolonialen tropischen Welt auf neue Art. Und der entscheidende Grund ist sicher nicht, wie E. Jünger in den eingangs zitierten Beobachtungen wahrzunehmen meinte, die ,geistiger und körperlicher Verheerung“, die die tropische Welt anrichtet. Der Grund ist die tiefe kulturelle Verankerung dieser Bildungsidee in die nördliche, die protestantische Welt. Diese Verankerung ist ihr sicher nicht vorzuwerfen, dafür aber ihr universeller Anspruch, der von einer Blindheit ihren eigenen Entstehungsbedingungen gegenüber zeugt. Damit läuft die Bildungsidee tatsächlich Gefahr, gegen ihre eigene Intention, zum Erfüllungsgehilfen imperialistischer Ideologie zu werden, wie es verabsolutierend Fernández VÁZQUES (2002; 2003) behauptet. $^{21}$ Gegen eine solche Verabsolutierung der Bildungsidee ist Macunaíma sicherlich immun. Allerdings schließt Mário de Andrade doch einen wohl von ihm selbst nicht wahrgenommenen Pakt mit dem Bildungsroman und zwar in seinem - zumindest anfänglichen - Vertrauen auf das Poetische und auf die Wirkungskraft des Ästhetischen. Als poetische Realisierung - darauf wurde oben hingewiesen - war er mit seinem Helden sehr einverstanden. Wie der Bildungsroman von der bildenden und erzieherischen Wirkung des Ästhetischen und der Institution, in der es seine umfassendste Verwirklichung findet, der Kunst, überzeugt war, so war auch Mário de Andrade von der „,aufklärerischen“ Wirkung seines Macunaíma überzeugt: Aufklärung durch Lachen, durch das Lachen des Leserpublikums über die Amoralität des Helden, die es seine eigenen Schwächen und Pakte mit dieser Amoralität wahrnehmen lässt.

\footnotetext{
${ }^{21}$ Siehe Kapitel 4: "El Bildungsroman postcolonial: reescribiendo la tradición” (2003: 115- 121).
} 
Nitschack, H. - Macunaíma, ein Wilhelm Meister in den Tropen?

Sein verzweifeltes ,ich bin gescheitert“ in dem Brief an Álvaro Lins schreibt Mário de Andrade auf dem Höhepunkt des „Estado Novo“ (1937-1945) unter der Regierung Getúlio Vargas', die trotz ihres militärischen, aber vor allem wirtschaftlichen Bündnisses mit den Vereinigten Staaten deutliche faschistische Züge trug. Mário de Andrade musste einsehen, dass es ihm nicht gelungen war, in seinem Roman eine Ästhetik zu entwickeln, von der ein Widerstand gegen diese faschistische Wende in der brasilianischen Geschichte hätte ausgehen können. Ja es sind die Falschen, die ihm jetzt applaudieren. Damit teilte er aber dann doch eine Hoffnung, oder eine Illusion mit dem Bildungsroman, der ebenso davon überzeugt war, dass er nicht nur die Bildungsgeschichte - die resignierte oder gescheiterte Bildungsgeschichte - seines Helden erzählen würde, sondern mehr noch, dass dieses Erzählen eine „bildende“ oder eine aufklärende Wirkung auf die Leser haben könnte.

Macunaíma als jüngeren Bruder Wilhelm Meisters zu lesen, eine Wahlverwandtschaft zwischen beiden zu behaupten, bezieht sich also nicht auf ihre gemeinsame Vergangenheit, da sind die beiden zu verschieden, sondern sie vereint eine gemeinsame Sehnsucht nach einer zukünftigen nationalen Kultur, in der die Subjektivität ihrer Bürger sich entfalten kann und die sie gleichzeitig fördert. Die Schwierigkeiten und Widerstände, die sich diesem Projekt entgegen stellen, sind unter den brasilianischen Bedingungen sicher viel schwerer zu überwinden als in der Welt Wilhelm Meisters. Darum scheint auch auch Mário de Andrade in seiner Korrespondenz an der Möglichkeit seiner Verwirklichung zu verzweifeln. Bei Wilhelm wird das familiäre Glück und die Aussicht, seinen Ort in dieser Gesellschaft zu finden, mit dem Tode Mignons und dem Selbstmord des Harfners erkauft, für Macunaíma scheint eine friedlichere und glücklichere Welt vorerst nur als Stern vorstellbar zu sein.

\section{Literaturverzeichnis:}

AlmeIDA, Maria Cândida Ferreira de. "Só me interessa o que não é meu": a antropofagia de Oswald de Andrade. http://www.globalcult.org.ve/doc/CandidaRelea.doc ANDERSON, Benedict. Imagined Communities. London, Verso, 1994 [1983]. 
Nitschack, H. - Macunaíma, ein Wilhelm Meister in den Tropen?

ANDRADE, Mário de. Macunaíma: o herói sem nenhum caráter. Edição Crítica. Telê Porto Ancona Lopez (Coord.). Madrid, Paris, México, São Paulo, Lima, Guatelmala, San José de Costa Rica, Santiago de Chile. Editorial Universitaria Chile (e.o.) Santiago de Chile: Colección Archivos, 1997 [1928].

. "Correspondência". In: ANDRADE, Mário de: Macunaíma: o herói sem nenhum caráter. Edição Crítica. Telê Porto Ancona Lopez (Coord.). Madrid, Paris, México, São Paulo, Lima, Guatelmala, San José de Costa Rica, Santiago de Chile. Editorial Universitaria Chile (e.o.) Santiago de Chile: Colección Archivos, 1997 [1928]

ANDRADE, Oswald de. "Pau Brasil" [1924] In: A utopia antropofágica. São Paulo, Editora Globo, 1990. pp. 41-45.

. “O manifesto antropófago" [1928] In: A utopia antropofágica. São Paulo, Editora Globo, 1990. pp. 47-55.

. “A Crise da Filosofia messiânica”, In: A utopia antropofágica. São Paulo, Editora Globo, 1990. pp. 101-159.

ANTElo, Raúl: "Canibalismo e diferença”. In: RochA, João Cezar de Castro y Ruffinelli, Jorge. Anthropophagy Today? Nuevo Texto Crítico, Enero-diciembre 1999, N²3/24, Stanford University, pp. 129-140.

Bitarães NetTo, Adriano. Antropofagia oswaldiana: um receituário estético e científico. São Paulo: Annablume, 2004.

BIRUS, Hendrik. Grösste Tendenz des Zeitalters. Oder Ein Candide, gegen die Poesie gerichtet? Friedrich Schlegels und Novalis' Kritik des Wilhelm Meister. http://www.goethezeitportal.de/db/wiss/goethe/meisterslehrjahre birus.pdf

Cavalcanti ProençA, Manoel. Roteiro de Macunaíma. Rio de Janeiro: Editora Civilização Brasileira: 1973 [1950]

DunN, Christopher. Brutality Garden: Tropicália and the Emergence of a Brazilian Counterculture. Chapel Hill: UNC Press, 2001.

EINSTEIN, Carl. Negerplastik. Verlag der Weißen Bücher (Kurt Wolff), Leipzig 1915.

FERNÁNDEZ VÁZQUES, José Santiago. La novela de formación. Una aproximación a la ideología colonial europea desde la óptica del Bildungsroman clásico. Alcalá de Henares, 2002.

. Reescrituras postcoloniales del Bildungsroman. Madrid: Ed. Verbum, 2003.

GoETHE, Johann Wolfgang. Wilhelm Meisters Lehrjahre. In: Goethes Werke, Bd. VII Hg. von Erich Trunz. München: Verlag C.H. Beck, 1981.

. Die Wahlverwandtschaften In: Goethes Werke, Bd. VI, Hg. von Erich Trunz. München: Verlag C.H. Beck, 1988. [1795/96]

HöLDERLIN, Friedrich. Hyperion. Hrsg. von Knaupp, Michael. Stuttgart: Reclam, 1997. [1797 und 1799]

Humboldt, Alexander von. Reise in die Aequinoctial-Gegenden des neuen Continents. Hrsg. von Hauff, Hermann. Stuttgart: Cotta 1859-1860.

JAuregui, Carlos A.. Canibalia. Canibalismo, calibanismo, antropofagia cultural y consumo en América Latina. Madrid, Frankfurt a. M: Vervuert: 2008.

Pandaemonium, São Paulo, v. 16, n. 22, Dez/2013, p. 156-178 
Nitschack, H. - Macunaíma, ein Wilhelm Meister in den Tropen?

JÜNGER, Ernst. Atlantische Fahrt. Tübingen, Otto Reichl Verlag, 1949 [1947. London]

KeCK, Anette / KORDING, Inka / PROCHASKA, Anja (eds.). Verschlungene Grenzen Anthropophagie in Literatur und Kulturwissenschaften. Literatur und Anthropologie, Tomo 2, Tübingen: Narr, 1999.

Keyserling, Hermann. Das Reisetagebuch eines Philosophen. Zwei Bände, Reichl, Darmstadt, 1919.

KOCH-GRÜNBERG, Theodor. Vom Roroima zum Orinoco. Ergebnisse einer Reise in Nordbrasilien und Venezuela in den Jahren 1911-1913. 1. Band, Berlin, Dietrich Reimer, 1917.

Koch-GRÜNBERG, Theodor: Vom Roroima zum Orinoco. 2. Band, Stuttgart, Strecker und Schröder, 1924.

LuKÀCS, Georg. Die Theorie des Romans. Berlin: Luchterhand, Neuwied, 1920. (http://www.gutenberg.org/files/26972/26972-h/26972-h.htm)

NICOLAI, Friedrich. „Vertraute Briefe von Adelheid B** an ihre Freundin Julie S**“, In: NICOLAI, Friedrich: Kritik ist überall, zumal in Deutschland, nötig. Satiren und Schriften zur Literatur. Hrsg. v. Wolfgang ALBRECHT, Leipzig/Weimar 1987, pp. 44-180. In: BIRUS, Hendrik. „Grösste Tendenz des Zeitalters. Oder Ein Candide, gegen die Poesie gerichtet? Friedrich Schlegels und Novalis' Kritik des Wilhelm Meister". http://www.goethezeitportal.de/db/wiss/goethe/meisterslehrjahre birus.pdf (15.11.2011)

NiTSCHACK, Horst. "Macunaíma und Serafim Ponte Grande: Literarische Dekomposition der Wirklichkeit als Subversion ihrer Macht." In: STROSETZKI, Christoph (Ed.): Zwischen Ideologisierung und Ausgrenzung. Diskurse der Herrschaft in Lateinamerika. Rheinfeld und Berlin: Schäuble, 1996. pp. 97-112.

. "O mito do índio no modernismo brasileiro e nas vanguardas hispanoamericanas." In: BERG, Walter Bruno, u. a.: As Américas do Sul: O Brasil no Contexto Latino-Americano. Beihefte zur Iberoromania Bd. 17. Tübingen: Max Niemeyer, 2001, pp. 108-118.

NUNES, Benedito. "O retorno à antropofagia." In: ROCHA, João Cezar de Castro y RUFFINELLI, Jorge. Anthropophagy Today? Nuevo Texto Crítico, Enero-diciembre 1999, № 23/24, Stanford University, pp. 231-234.

. "Antropofagia ao alcance de todos". In: ANDRADE, Oswald: A utopia antropofágica. São Paulo, Globo: 1990. pp. 5 - 39.

RochA, João Cézar de Castro y RUFFINELLI, Jorge. Anthropophagy Today? Nuevo Texto Crítico, Enero-diciembre 1999, N²3/24, Stanford University.

SCHLEGEL, Friedrich. Athenäum. Kritische Friedrich-Schlegel-Ausgabe, Bd. 2. München/Paderborn/Wien u. Zürich: Schöningh, 1967.

SCHWARTZ, Jorge. Vanguardas latino-americanas; polémicas, manifestos e textos críticos. São Paulo: Edusp, Iluminuras, Fapesp, 1995

SCHWARZ, Roberto. "Idéias fora do lugar". In: SCHWARZ, Roberto. Ao vencedor as batatas. São Paulo: Livraria Duas Cidades, 1992. pp. 13-28.

SEvcenko, Nicolau. Orfeu extático na Metrópole. São Paulo, sociedade e cultura nos frementes anos 20. São Paulo: Compahia das Letras, 1992.

Pandaemonium, São Paulo, v. 16, n. 22, Dez/2013, p. 156-178 
Nitschack, H. - Macunaíma, ein Wilhelm Meister in den Tropen?

SOMMER, Doris. Fondational ficitions: the national romances of Latin America. Oxford; Berkeley; Los Angeles: Univ. of California Press, 1991.

SPENGLER, Oswald. Der Untergang des Abendlandes. 2 Bde. München, C.H. Beck'sche Verlagsbuchhandlung 1929.

STADEN, Hans von. Warhaftige Historia und beschreibung eyner Landtschafft der wilden nacketen grimmigen Menschenfresser Leuthen in der Newenwelt America gelegen Kritische Ausg. I Franz Obermeier. Übertr. ins heutige Dt.: Joachim Tiemann. Trad. ao portug.: Guiomar Carvalho Franco. - [Nachdr. der Ausg. Marburg 1557] Kiel: Westensee Verlag 2007.

recebido em 18/04/2013

aceito em 31/08/2013 